\title{
Local effects of adipose tissue in psoriasis and psoriatic arthritis
}

\author{
This article was published in the following Dove Press journal: \\ Psoriasis: Targets and Therapy \\ 3 February 2017 \\ Number of times this article has been viewed
}

\author{
Ilja L Kruglikov' \\ Uwe Wollina ${ }^{2}$ \\ 'Scientific Department, Wellcomet \\ GmbH, Karlsruhe, ${ }^{2}$ Department of \\ Dermatology and Allergology, Hospital \\ Dresden-Friedrichstadt Academic \\ Teaching Hospital of the Technical \\ University of Dresden, Dresden, \\ Germany
}

\begin{abstract}
The structure and physiological state of the local white adipose tissue (WAT) located underneath the lesional psoriatic skin and inside of the joints affected by psoriatic arthritis play an important role in the pathophysiology of these diseases. WAT pads associated with inflammatory sites in psoriasis and psoriatic arthritis are, correspondingly, dermal WAT and articular adipose tissue; these pads demonstrate inflammatory phenotypes in both diseases. Such local WAT inflammation could be the primary effect in the pathophysiology of psoriasis leading to the modification of the local expression of adipokines, a change in the structure of the basement membrane and the release of keratinocytes with consequent epidermal hyperproliferation during psoriasis. Similar articular adipose tissue inflammation can lead to the induction of structural modifications and synovial inflammation in the joints of patients with psoriatic arthritis.
\end{abstract}

Keywords: psoriasis, psoriatic arthritis, pathophysiology, adipose tissue, dermal adipocytes, articular adipose tissue

\section{Introduction}

Psoriasis and psoriatic arthritis (PsA) are actually considered to be interrelated diseases that share some pathophysiologic pathways. ${ }^{1}$ One important argument for this is that they exhibit similarities in prevalence statistics: while inflammatory arthritis occurs in $2 \%-3 \%$ of the general population, its prevalence among psoriatic patients is much higher. The prevalence of PsA also shows similar ethnic variations to those of psoriasis; the prevalence of PsA is high in Caucasians from Europe and low in Asians. ${ }^{2}$

There is growing evidence that both diseases are related to the physiologic state of the adipose tissue. ${ }^{3}$ Whereas such a relationship was normally considered to exist at the systemic level, it was shown that some inflammatory skin diseases demonstrate a reciprocal expression of inflammatory and lipid genes in lesional skin. ${ }^{4}$ Psoriatic patients have an altered composition of high-density lipoproteins in serum and an impaired cholesterol efflux from macrophages, ${ }^{5}$ and antipsoriatic therapy is capable of restoring the normal balance. ${ }^{6,7}$ Also, there is a high prevalence of metabolic syndrome (MetS) in patients with psoriasis and $\mathrm{PsA}^{8}$; furthermore, psoriasis and PsA patients with MetS have significantly higher psoriasis area and severity index (PASI) values than the patients without MetS. ${ }^{8-10}$ These are the important arguments for the systemic involvement of adipose tissue in the pathology of psoriasis.

At the same time, skin inflammation in patients with psoriasis is restricted to psoriatic plaques, and thus, its appearance is generally spatially limited. This observation supports the primary local modification of the superficial layer of the subcutaneous 
white adipose tissue (sWAT) underneath the psoriatic plaques. ${ }^{11,12}$ According to this theory, adipose tissue interacts with the skin not only at a systemic level, which should be mainly connected to the function of abdominal sWAT, but also with the skin locally, producing the expansion and/or physiologic modification of dermal WAT (dWAT), which leads to the overproduction of the antimicrobial peptide (AMP) cathelicidin in dermal adipocytes. ${ }^{13}$

Very recently, the expression of the microRNA (miR) member miR-26b-5p was shown to be strongly upregulated locally in sWAT underneath the lesional psoriatic skin. ${ }^{14}$ This is especially interesting since miR-26b-5p downregulates cholesterol efflux from monocytes/macrophages, adipocytes, vascular endothelial cells and fibroblasts, which is in line with the results obtained by Holzer et al. ${ }^{5}$ The upregulation of miR-26b is connected to adipocyte differentiation, ${ }^{15}$ which was associated with inflammation in human WAT. Indeed, the absolute values of miR-26b are dependent on the number of macrophages that infiltrate a given fat depot, ${ }^{16}$ and miR-26b was shown to be regulated by different adipokine ${ }^{17}$ known to be produced in patients with psoriasis and PsA. Importantly, the expression of miRNA is strongly modified after immune stimulation, and miRNA was demonstrated to be able to alter the expression of cell surface proteins involved in the immune response in epithelial cells. ${ }^{18}$ Although a direct correlation between miR-26b expression and cathelicidin production in adipose tissue was not investigated, such a relationship is strongly assumed to exist, especially because cathelicidin is also expressed by macrophages.

Analogous to psoriasis, it can be supposed that PsA is connected to a pathologic modification of the articular adipose tissue (AAT) in an affected joint. From this point of view, the systems epidermis-dermis-sWAT in psoriasis and the synovium-AAT in PsA should demonstrate some structural and physiologic similarities. This shifts the main emphasis from systemic to local effects of adipose tissue in psoriasis and PsA. In this review, we discuss the recent insights in this field.

\section{Adipose tissue in psoriasis}

Psoriasis is actually considered to be a systemic inflammatory disease with a heterogeneous genetic background characterized by the dysregulation of the immune system, an impaired barrier function and epidermal hyperproliferation. It is broadly accepted that various cytokines are involved in the pathophysiology of this disease, which include interleukin (IL)-17, IL-22 and IL-23. ${ }^{19-21}$ At the same time, some authors have stated that IL-17 causes no psoriasis-like morphologic changes in the skin. ${ }^{22}$ Recently, it was shown that obesity can exacerbate imiquimod-induced psoriasiform dermatitis in mice by the upregulation of IL-17A, IL-22 and regenerating islet-derived protein 3 gamma (Reg3 $\gamma$ ) expression. ${ }^{23}$ It should be noted that Reg $3 \gamma$ not only enhances keratinocyte proliferation, but is also an adenosine monophosphate (AMP) that demonstrates a strong reaction against Staphylococcus aureus. $^{24}$

The pathophysiology and inflammatory nature of psoriasis have been connected with the physiologic state of the systemic WAT, especially with the intensity and spectrum of secreted adipokines, ${ }^{25}$ resulting in the unbalanced production of pro- and anti-inflammatory signaling proteins. ${ }^{26}$ This finding supports the opinion that WAT is a key participant in the development of different skin pathologies, ${ }^{27}$ including psoriasis.

\section{Systemic levels of adipokines and psoriasis}

Different adipokines demonstrate various activities in patients with psoriasis. For example, the secretion of tumor necrosis factor (TNF)- $\alpha$ and IL-6 from keratinocytes as well as the secretion of IL-6, IL-8, IL-17, IL-22 and TNF- $\alpha$ from T-lymphocytes can be stimulated by leptin and inhibited by adiponectin. ${ }^{28}$ At the same time, different authors have reported very inconsistent levels of adipokines such as adiponectin and leptin in the serum of psoriatic patients. ${ }^{29}$ Whereas high serum levels of leptin were found in some clinical studies of patients with psoriasis, ${ }^{29,30}$ other studies reported significantly reduced levels of this adipokine, which were also strongly dependent on PASI. ${ }^{31}$ Adiponectin is primarily known as a metabolic regulator of insulin sensitivity and a protector against inflammation in MetS. ${ }^{32}$ Its anti-inflammatory properties were demonstrated, among others, in AdipoR1/AdipoR2-double-knockout (KO) mice. ${ }^{33}$ Serum levels of adiponectin and IL-22 were reported to be positively correlated in patients with psoriasis. ${ }^{34}$

The importance of adiponectin in the pathophysiology of psoriasis became evident after it was shown that this adipokine was highly involved in the pathophysiology of IL-17-related psoriasiform dermatitis and that the adiponectin $\mathrm{KO}$ mice demonstrated a severe form of this disease. ${ }^{35}$ Moreover, application of exogenous adiponectin significantly suppressed the elevated production of IL-17 in these mice and improved the cutaneous lesions. Importantly, exogenous adiponectin did not suppress IL-17 expression in wild-type mice, which means that the mechanism of such a suppression should have a threshold character. It should be taken into 
account that psoriasiform dermatitis in adiponectin $\mathrm{KO}$ mice was induced by the topical application of imiquimod, ${ }^{35}$ which is known to cause an almost complete depletion of the dWAT layer in murine skin. ${ }^{35,36}$ Such a depletion can significantly reduce the local reaction of WAT underneath the lesional skin, thus reducing the local immune response of the skin to pathogens and increasing the inflammatory reaction induced by these pathogens.

The adiponectin content in patients with psoriasis was mainly measured in serum samples, which reflects the systemic level of this adipokine, and it was found to be significantly higher in PsA patients than in psoriatic patients without arthritis. ${ }^{8}$ This can at least partly explain the inconsistent results obtained by different authors who did not differ between these groups. ${ }^{29}$ Moreover, adiponectin demonstrated a negative correlation and leptin a positive correlation with body mass index, and both adipokines correlated with PASI. ${ }^{31}$ Interestingly, serum adiponectin was significantly decreased in patients with mild (PASI < 10) and moderate $(10<$ PASI $<20)$ psoriasis, but it almost reached control values in patients with severe (PASI > 20) psoriasis. Although antipsoriatic treatments can significantly reduce PASI values, they do not affect the serum levels of both adipokines. ${ }^{37}$ These results indicate that skin appearance in patients with psoriasis is not primarily connected to the systemic levels of adipokines.

Another parameter that can sufficiently influence the levels of adipokines in serum and was not properly controlled in the majority of psoriatic studies is the presence of MetS. Indeed, the concentration of serum adiponectin was found to be significantly reduced in controls with MetS and in psoriasis patients with MetS, compared to controls without MetS. At the same time, serum leptin was significantly higher in psoriasis patients with MetS than in psoriasis patients without MetS. ${ }^{10}$ In this study, the serum concentration of IL-6 was not significantly different from the control values. This could mean that MetS influences serum adiponectin and leptin levels to a greater extent than psoriasis, which must be taken into account in the interpretation of experimental and clinical results. It should also be noted that the coefficients of variation for all investigated adipokines in every group were very high, which normally means that these groups were not homogeneous and that there should be additional hidden parameters to define the adipokine levels in serum. ${ }^{10}$

\section{Local levels of adipokines in sWAT in patients with psoriasis}

The same authors also investigated the local adipokine levels in sWAT underneath the lesional psoriatic skin. ${ }^{10}$ They found that the local concentrations of adiponectin and TNF- $\alpha$ in sWAT were not statistically different between controls and the groups with psoriasis and/or MetS. The local concentration of leptin in sWAT was not different between the psoriasis group and controls, but significantly differed between the groups of psoriasis patients with and without MetS. ${ }^{10}$ Also, the local concentration of IL-6 in sWAT underneath the lesional psoriatic skin was significantly higher in controls than in patients with psoriasis. Fourteen out of 18 patients were treated with topical corticosteroids and vitamin D analogs, which were interrupted before the biopsy. This could have shifted the local concentrations of adipokines in sWAT at the moment of biopsy, compared with their levels in untreated patients.

Concurrently, another group revealed that the local adiponectin levels in human skin and in sWAT underneath the skin lesions were significantly lower in patients with psoriasis than in control subjects. ${ }^{35}$ This is in contrast to the results obtained by Mitsuyama et al, ${ }^{10}$ but correlates well with the anti-inflammatory properties of adiponectin and with the hypothesis of a local, not systemic, connection between adiponectin and psoriasis. $^{11}$

\section{Adiponectin in innate immunity}

Adiponectin was also recognized as a regulator of innate immunity. ${ }^{38}$ This function of adiponectin correlates with recent observations that dermal adipocytes are involved in the innate skin protection against infection. ${ }^{39}$ Infection of murine skin with $S$. aureus induced a quick proliferation of preadipocytes and a massive expansion of dermal adipose tissue thickness. At the same time, mice with impaired adipogenesis demonstrated reduced protection against $S$. aureus infection. Such a defense function was mediated through the high-level production of AMPs related to cathelicidin that were released by dermal adipocytes during early adipogenesis. From the above, it can be predicted that such an immune reaction should be strongly reduced in the case of psoriasiform dermatitis induced by imiquimod, ${ }^{35}$ since the dWAT is almost completely depleted in this model.

Taken together, we can note the growing evidence that skin inflammation in psoriasis is connected with the function of the adipose tissue. Such a connection appears to be primarily realized through a local interaction between the epidermis and underneath the dWAT layer.

\section{Adipose tissue in PsA}

Traditionally, psoriasis and PsA are considered to be autoimmune diseases that share some pathophysiologic mechanisms involving T-cell reactions and differ mainly in their targets, 
which are skin and synovial tissue, respectively. Contrarily, it was proposed that enthesopathies (disorders involving the attachment of tendons or ligaments to a bone), not an autoimmune reaction, could be the primary reason for PsA. ${ }^{40}$ Indeed, enthesitis (together with peripheral arthritis, axial disease and dactylitis) belongs to a typical manifestation of PsA, and it was even stated that PsA was more similar to undifferentiated spondyloarthropathy than to rheumatoid arthritis. ${ }^{41}$ Because of this, our discussion of the role of adipose tissue in PsA will take into account both synovial tissue and entheses.

\section{Structure of AATs}

The main joints known to be affected by PsA contain the localized fat pads (AATs) such as the Hoffa's (infrapatellar) fat pad (IPFP) in the knee, the heel fat pad (HFP) or the anterior and posterior fat pads in the elbow. The adipose tissue is also localized on the tendon surfaces and in the bursa.

The IPFP is the AAT within the knee joint, which was investigated more in depth than other AATs. The IPFP was recently histologically described as SWAT of the lobular type with lobules covered by thick fibrous sheaths that produced baskets around the cells. In the IPFP, the lobules appear smaller and the fibrillary structure appears thicker than in the abdominal sWAT. ${ }^{42}$ This structure corresponds to the "fibrous" type of adipose tissue as described by Sbarbati et $\mathrm{al}^{43}$ and Bertossi et al. ${ }^{44}$ The HFP was also described as a "fibrous" AAT with small lobules of adipocytes covered by thick distinct fibrous shells. ${ }^{45}$

The "fibrous" type of WAT, which appears to be typical for AATs, has better mechanical and, thus, protective properties than the "structural" type of WAT that contains less collagen around the adipocytes. ${ }^{46}$ At the same time, the "fibrous" WAT normally contains smaller adipocytes and should be more prone to inflammation. ${ }^{47}$

\section{AATs as active arthritis units}

It was proposed that the IPFP should be considered as an active osteoarthritic joint tissue that can secrete interleukins and adipocytokines. ${ }^{48}$ It was also stated that the IPFP has an inflammatory phenotype in patients with osteoarthritis and rheumatoid arthritis. ${ }^{49-52}$ Indeed, the IPFP volume positively correlates with knee cartilage volume and negatively correlates with the structural abnormalities in the synovium, which indirectly supports the anti-inflammatory role of the IPFP in knee osteoarthritis. ${ }^{53,54}$ Interestingly, resection of the juxta-articular adiposis dolorosa (lipohypertrophy of the inner or outer parts of the knee joints) significantly reduced or even fully eliminated the joint pain. ${ }^{55}$
The HFP structure in rheumatoid arthritis and in spondyloarthropathies was also shown to be strongly modified, whereas this modification occurs more frequently in an inflammatory-edematous and less frequently in a degenerative-atrophic pattern, ${ }^{56}$ which means that foot arthritis correlates with inflammation in the HFP. It is, however, still not clear whether this is a primary or secondary effect of synovial inflammation and arthritis.

\section{Role of adipose tissue in enthesopathies}

Adipose tissue is also a common feature of normal enthesis, where it appears in enthesis itself as well as in the angle between the tendon/ligament and the bone. One of the most prominent adipose tissue pads that are associated with enthesis is the Karger's (retromalleolar) fat pad (KFP) in the Achilles area. The KFP is composed of unilocular adipocytes that are separated by small bundles of elastic and collagen (type I) fibers that produce a structure similar to that of the HFP. ${ }^{57}$

Adipose tissue was supposed to be involved in the pathophysiology of enthesopathies, similar to the involvement of the IPFP and HFP in joint inflammation in the knee and heel, respectively. Recently, it was demonstrated that KFP has an inflammatory phenotype and an altered lipid metabolism in patients with Achilles tendinopathy, compared with healthy controls. ${ }^{58}$ Adipocytes from such an inflammatory fat pad exhibit reduced lipolysis and adiponectin secretion but increased fatty acid $\beta$-oxidation.

Moreover, it was proposed that the inflammatory reaction in enthesis can be the primary reason for PsA. ${ }^{40}$ This inflammation can be caused by microtrauma, which is similar to the Koebner phenomenon in patients with psoriasis. Also, such inflammation can be caused by a proinflammatory reaction of the surrounding adipose tissue..$^{59}$

\section{Adipokine production in AATs}

AATs can express different adipokines with significantly different concentrations in controls and in subjects with arthritis. ${ }^{60}$ It was shown that the IPFP from osteoarthritic patients expresses higher levels of leptin and chemerin compared with healthy controls, ${ }^{61}$ rheumatoid IPFP secretes both pro- (IL-6, IL-8 and TNF) and anti-inflammatory (IL-1Ra) cytokines as well as the classical adipokines, ${ }^{62}$ and the expression of leptin and adiponectin increases with the progressing stage of osteoarthritis. ${ }^{63}$ Recently, it was also demonstrated that the WNT1 inducible signaling pathway protein 2 in the IPFP is significantly increased in patients with osteoarthritis compared with control subjects. ${ }^{64}$ This adipokine can be involved in the crosstalk between the IPFP and the synovium, 
since it regulates preadipocyte commitment and activates the master adipogenic factor peroxisome proliferator-activated receptor gamma, ${ }^{65}$ which is essential for the production of mature adipocytes.

The role of adiponectin in joint diseases remains contradictive. Adiponectin was thought to have a protective effect in osteoarthritis. ${ }^{66}$ Adiponectin levels in plasma and synovial fluid decrease significantly with the increased severity of osteoarthritis, which can indeed suggest a protective role of adiponectin in this disease. ${ }^{67}$ On the other hand, it was stated that adiponectin is involved in key pathways of inflammation and in matrix degradation in the joints. ${ }^{68}$

\section{AMPs in psoriasis and PsA}

The expression of AMPs was incorporated into the pathogenesis of different autoimmune diseases. ${ }^{69,70}$ Such expression was investigated both in patients with psoriasis and PsA ${ }^{71}$ and was found to be different: whereas a broad spectrum of AMPs was detected in psoriatic skin, only some of these peptides were found in the synovium of PsA patients. ${ }^{72}$

Cathelicidin (LL-37) generally demonstrates a proinflammatory effect; it attracts neutrophils, monocytes, T-cells and mast cells, and it is known to be strongly upregulated in psoriatic skin. ${ }^{73,74}$ At the same time, nonlesional skin in psoriasis demonstrates a trend toward increased expression of cathelicidin, compared to healthy skin. ${ }^{73}$ Moreover, nonlesional psoriatic skin also exhibits the same upregulation of cathelicidin expression after barrier disruption as the original psoriatic lesions, ${ }^{75}$ which means that this reaction should be of a local and not a systemic nature. In this context, topical application of calcipotriol, which leads to significant reduction of IL-17 and a decrease in cutaneous inflammation, correlates with a sufficient increase of cathelicidin expression in psoriatic plaques. ${ }^{73}$

It was proposed that the cathelicidins are involved in the pathogenesis of arthritis, since they were shown to be strongly upregulated in synovial membranes and in joints from humans and rats with rheumatoid arthritis. ${ }^{69,71}$ Interestingly, there are strong indications that vitamin D induces cathelicidin production and, thus, promotes innate immunity. ${ }^{76,77}$

The connection between cathelicidin and adipose tissue may be much more intriguing than was previously thought. Very recently it was shown that cathelicidin can suppress lipid accumulation in adipocytes by the inhibition of the CD36 receptor, ${ }^{78}$ which indicates that this peptide is a modulator of adipogenesis. Indeed, serum cathelicidin protein levels were found to be significantly increased in nondiabetic obese patients compared with nondiabetic controls with normal body mass index values. Since the CD36 receptor not only facilitates the transport of long-chain fatty acids into cells but also binds many ligands including collagens, ${ }^{79}$ the production of cathelicidin in adipocytes ${ }^{39}$ can modulate not only the lipid content in adipocytes but also the extracellular matrix (ECM) in WAT. This means that the pathogens can modulate the whole structure of sWAT, especially of the superficial sWAT layer, dWAT.

\section{Role of collagen $\mathrm{VI}$ in psoriasis and PsA}

The ECM contains different types of collagen, including collagen type VI (COL6), which is known to be involved in various regulatory processes in the adipose tissue, bones, cartilage and skin. ${ }^{80}$ Different chains of COL6 are distinctly present in the skin. Whereas COL6a5 (also known as COL29a1) is present in the epidermis, COL6a3 is not found there. However, it is distributed throughout the dermis and is particularly abundant in the basement membrane at the epidermal-dermal interface. ${ }^{81}$ This interface is connected with the dermal ECM through a protein network containing COL1, COL4, COL5a1, COL6a3 and laminin-111. ${ }^{82}$

Proteogenomic analysis revealed that the expression levels of COL1a1, COL1 22 and COL6a 3 are strongly reduced both at the mRNA and the protein levels in papillary and reticular dermis from psoriatic plaques, compared with corresponding values in the nonlesional skin of psoriatic patients. ${ }^{83}$ Such a reduced expression of collagens in the lesional psoriatic skin should evidently impair the mechanical stability of the basement membrane and release the keratinocytes that are anchored to its opposite side. Both the hyperproliferation of keratinocytes and the structural modifications of the basement membrane in psoriatic plaques (splitting, excessive folding and production of large gap areas with reduced collagen content) are known as typical pathologic signs of psoriatic skin.

Whereas the expression of COL6 in the skin is mainly connected with fibroblasts, ${ }^{80}$ it is very doubtful that this process is fully independent of the state of COL6 in the underlying sWAT. First, sWAT is the most abundant source of COL6; it produces the pericellular fibrous structures around adipocytes. ${ }^{84}$ Consequently, its expression should be stronger in the "fibrous" type WAT. Expression of COL 6 by the adipocytes cannot be spatially restricted only to sWAT and should be noticeable in the dermis at least several cell diameters away from the dermis/sWAT interface. Moreover, while dermal adipocytes can penetrate deep into the dermis 
and in some cases, can even reach the papillary dermis, this induction of COL 6 should be present in a significant part of the dermis volume.

Second, COL6 not merely exerts structural functions, but can also act as a paracrine factor. This effect was shown in the process of mammary tumorigenesis. ${ }^{85}$ It should be noted that monocyte-derived macrophages are able to produce COL6 in a nonfibrillar form, which cannot be incorporated into the ECM, but appears to mediate cell-cell interactions and cell adhesions. ${ }^{86}$

Third, there is an interaction between fibroblasts and adipocytes from the underlying sWAT, which can significantly modulate the synthetic activity of these skin cells. ${ }^{87}$ From this point of view, collagen arrangement in the dermis and in the underlying sWAT should be considered as a single network, which additionally strengthens the role of the local sWAT in psoriasis.

Also, COL6 is involved in cutaneous innate immunity and different pathogens exhibit strong affinity for this collagen. ${ }^{88}$ COL6 has a very low triple helix content and is predominantly composed of globular von Willebrand factor type A domains. ${ }^{89}$ Peptides from these domains are able to display a dose-dependent antimicrobial activity. ${ }^{90}$

Very recently, it was demonstrated that COL6a3 knockdown in adipocytes leads to inflammatory resistance by the suppression of monocyte chemoattractant protein 1, which points to an interconnection between COL6 and inflammation. ${ }^{91}$ While systemic modification of COL6 content was shown to be generally connected with MetS, ${ }^{92}$ local modification of the adipose tissue ECM can be assumed to be significant in some skin pathologies. It was also shown that cathelicidin regulates myeloid cell accumulation in WAT and can thus promote insulin resistance. ${ }^{93}$ The realization of such a reaction locally in sWAT underneath the lesional psoriatic skin could mean the occurrence of a local pathologic condition corresponding to the appearance of the local "insulin resistive area". In this context, it is noteworthy that patients with PsA but not rheumatoid arthritis have a significantly higher prevalence of MetS. ${ }^{94}$ As mentioned above, this prevalence is also higher in patients with PsA than in patients with psoriasis. $^{8}$

Chondrocytes are also encapsulated by pericellular fibrosis, which in normal cartilage consists mainly of COL $6 .{ }^{95}$ It was shown that aged COL6alKO mice demonstrate progressive osteoarthritic joint degeneration. ${ }^{96}$ The lack of COL6 results in a loss of stiffness of the articular cartilage, which correlates with the results obtained in a previous study ${ }^{92}$ on the mechanical modules of WAT. Remarkably, strongly dysfunctional fibrillogenesis was found in the tendons of COL6a1KO mice. ${ }^{97}$

In summary, COL6 - the most important structural component of ECM in the adipose tissue - appears to be involved in a crosstalk between superficial WAT and the epidermis/ papillary dermis. This collagen also influences the structure and stability of the protein network that bridges the basement membrane and the dermal ECM. On the other side, adipocytes should be considered as cells having a double participation in cutaneous innate immunity through COL6 expression and cathelicidin production. ${ }^{39}$ Future research should examine whether these two processes are interconnected.

\section{Interaction of epidermal and dermal cells with adipocytes}

The presence of spatially limited hyperproliferative epidermal areas in patients with psoriasis that are connected to the local modification of the superficial adipocytes located near the interface of the dermis/subcutis strongly suggests that these adipocytes must have an ability to influence fibroblasts and keratinocytes and induce their proliferation. ${ }^{11,98}$

Adipocytes can interact with fibroblasts and affect their synthetic function, and this interaction appears to be dependent on the adipocyte size. It was shown that enlarged adipocytes can significantly suppress the synthetic activity of co-cultured fibroblasts, whereas small adipocytes failed to do so. ${ }^{87}$ Adiponectin expression in adipocytes is also cell size dependent and is higher in smaller adipocytes, ${ }^{99,100}$ which should be more typical for "fibrous" type WAT. ${ }^{44}$ On the other hand, such an interaction in vivo can be realized through paracrine signaling only for the neighboring areas that contain fibroblasts and adipocytes, thus making the dWAT adipocytes the first choice for such a process.

The interaction of adipose tissue with skin cells is not limited to fibroblasts. Whereas IL-22 does not affect skin fibroblasts, endothelial cells or adipocytes, it can indeed interact with keratinocytes. ${ }^{22}$ Moreover, human keratinocytes can express adiponectin receptors, and adiponectin promotes both the proliferation and migration of these cells. ${ }^{27}$ Recently, it was shown that sWAT contains keratinocyte progenitor cells. $^{101}$

Thus, adipocytes from the superficial sWAT layer can interact with the epidermal and dermal cells to modulate their synthetic activity and proliferation.

\section{Conclusion}

Local inflammation in WAT is an important step in the pathophysiology of psoriasis and PsA. dWAT is the nearest 
to the epidermis adipose tissue depot and is the source of adipokines, keratinocyte progenitors, COL6 and AMPs, which are involved in the pathophysiology of psoriasis. Local production of adipokines and cathelicidin in dWAT underneath the lesional psoriatic skin can stimulate and regulate the local inflammatory cutaneous reactions. The production of COL6 can modulate the structure of the protein network that connects the basement membrane with the dermal ECM; the weakening of this structure influences the anchoring of keratinocytes and their release, thus contributing to epidermal hyperproliferation and modifications of the basement membrane that are typical in psoriasis. Similar processes should take place in PsA, whereas instead of dWAT, the corresponding AATs inside of the joints serve as the local sources of adipokines, AMPs and possibly COL6.

\section{Acknowledgment}

No specific funding was received from any bodies in the public, commercial or not-for-profit sectors to prepare this review.

\section{Disclosure}

ILK is the managing partner of Wellcomet $\mathrm{GmbH}$; no methods or devices of Wellcomet $\mathrm{GmbH}$ were used in this publication. UW reports no conflicts of interests in this work.

\section{References}

1. Yamamoto M, Nakajima K, Takaishi M, et al. Psoriatic inflammation facilitates the onset of arthritis in a mouse model. J Invest Dermatol. 2015;135(2):445-453.

2. Chandran V, Raychaudhuri SP. Geoepidemiology and environmental factors of psoriasis and psoriatic arthritis. J Autoimmun. 2010;34(3):J314-J321.

3. Toussirot E, Aubin F, Dumoulin G. Relationships between adipose tissue and psoriasis, with or without arthritis. Front Immunol. 2014;5:368.

4. Sääf AM, Tengvall-Linder M, Chang HY, et al. Global expression profiling in atopic eczema reveals reciprocal expression of inflammatory and lipid genes. PLoS One. 2008;3(12):e4017.

5. Holzer M, Wolf $\mathrm{P}$, Curcic $\mathrm{S}$, et al. Psoriasis alters HDL composition and cholesterol efflux capacity. J Lipid Res. 2012;53(8):1618-1624.

6. Holzer M, Wolf P, Inzinger M, et al. Anti-psoriatic therapy recovers high-density lipoprotein composition and function. J Invest Dermatol. 2014;134(3):635-642.

7. Marsche G, Holzer M, Wolf P. Antipsoriatic treatment extends beyond the skin: recovering of high-density lipoprotein function. Exp Dermatol. 2014;23(10):701-704.

8. Eder L, Jayakar J, Pollock R, et al. Serum adipokines in patients with psoriatic arthritis and psoriasis alone and their correlation with disease activity. Ann Rheum Dis. 2013;72(12):1956-1961.

9. Armstrong AW, Harskamp CT, Armstrong EJ. Psoriasis and metabolic syndrome: a systematic review and meta-analysis of observational studies. J Am Acad Dermatol. 2013;68(4):654-662.

10. Mitsuyama S, Higuchi T, Abe F, et al. Adipokines in subcutaneous adipose tissue are associated with metabolic syndrome in psoriasis patients. Toho J Med. 2015;1(1):7-13.

11. Kruglikov IL, Scherer PE, Wollina U. Are dermal adipocytes involved in psoriasis? Exp Dermatol. 2016;25(10):812-813.
12. Brembilla NC, Boehncke WH. Dermal adipocytes' claim for fame in psoriasis. Exp Dermatol. Epub 2016 May 19.

13. Kruglikov IL, Scherer PE. Dermal adipocytes: from irrelevance to metabolic targets? Trend Endocrin Metab. 2016;27(1):1-10.

14. Cheung L, Fisher RM, Kuzmina N, et al. Psoriasis skin inflammationinduced microRNA-26b targets NCEH1 in underlying subcutaneous adipose tissue. J Invest Dermatol. 2016;136(3):640-648.

15. Song $\mathrm{G}, \mathrm{Xu} \mathrm{G}, \mathrm{Ji} \mathrm{C}$, et al. The role of microRNA-26b in human adipocyte differentiation and proliferation. Gene. 2014;533(2):481-487.

16. Arner P, Kulyté A. MicroRNA regulatory networks in human adipose tissue and obesity. Nat Rev Endocrinol. 2015;11(5):276-288.

17. Xu G, Ji C, Shi C, et al. Modulation of hsa-miR-26b levels following adipokine stimulation. Mol Biol Rep. 2013;40(5):3577-3582.

18. Liu J, Drescher KM, Chen XM. MicroRNAs and epithelial immunity. Int Rev Immunol. 2009;28(3-4):139-154.

19. Sabat R, Ouyang W, Wolk K. Therapeutic opportunities of the IL22-IL-22R1 system. Nat Rev Drug Discov. 2014;13(1):21-38.

20. Bartlett HS, Million RP. Targeting the IL-17-TH17 pathway. Nat Rev Drug Discov. 2015;14(1):11-12.

21. Furue M, Kadono T. Psoriasis: behind the scenes. $J$ Dermatol. 2016;43(1):4-8.

22. Wolk K, Haugen HS, Xu W, et al. IL-22 and IL-20 are key mediators of the epidermal alterations in psoriasis while IL-17 and IFN- $\gamma$ are not. J Mol Med (Berl). 2009;87(5):523-536.

23. Kanemaru K, Matsuyuki A, Nakamura Y, Fukami K. Obesity exacerbates imiquimod-induced psoriasis-like epidermal hyperplasia and interleukin-17 and interleukin-22 production in mice. Exp Dermatol. 2015;24(6):436-442.

24. Choi SM, McAleer JP, Zheng M, et al. Innate Stat3-mediated induction of the antimicrobial protein Reg $3 \gamma$ is required for host defense against MRSA pneumonia. $J$ Exp Med. 2013;210(3):551-561.

25. Dalamaga M, Papadavid E. Adipocytokines and psoriasis: insights into mechanisms linking obesity and inflammation to psoriasis. World $J$ Dermatol. 2013;2(4):27-31.

26. Coimbra S, Catarino C, Santos-Silva A. The triad psoriasis-obesity-adipokine profile. J Eur Acad Dermatol Venereol. 2016; 30(11):1876-1885.

27. Shibata S, Tada Y, Asano Y, et al. Adiponectin regulates cutaneous wound healing by promoting keratinocyte proliferation and migration via the ERK signaling pathway. J Immunol. 2012;189(6): 3231-3241

28. Takahashi H, Honma M, Ishida-Yamamoto A, Iizuka H. Adiponectin and leptin modulate cell proliferation and cytokine secretion of normal human keratinocytes and T lymphocytes. J Dermatol Sci. 2010;59(2):143-145.

29. Gerdes S, Rostami-Yazdi M, Mrowietz U. Adipokines and psoriasis. Exp Dermatol. 2011;20(2):81-87.

30. Xue Y, Jiang L, Cheng Q, et al. Adipokines in psoriatic arthritis patients: the correlations with osteoclast precursors and bone erosions. PLoS One. 2012;7(10):e46740.

31. Baran A, Flisiak I, Jaroszewicz J, Świderska M. Effect of psoriasis activity on serum adiponectin and leptin levels. Postep Dermatol Alergol. 2015;32(2):101-106.

32. Ye R,Scherer PE. Adiponectin, driver or passenger on the road to insulin sensitivity? Mol Metab. 2013;2(3):133-141.

33. Iwabu M, Okada-Iwabu M, Yamauchi T, Kadowaki T. Adiponectin/ adiponectin receptor in disease and aging. NPJ Aging Mech Dis. 2015;1:15013.

34. Nakajima H, Nakajima K, Tarutani M, Sano S. Clear association between serum levels of adipokines and T-helper 17-related cytokines in patients with psoriasis. Clin Exp Dermatol. 2013;38(1):66-70.

35. Shibata S, Tada Y, Hau CS, et al. Adiponectin regulates psoriasiform skin inflammation by suppressing IL-17 production from $\gamma \delta$-T cells. Nat Commun. 2015;6:7687.

36. van Belle AB, de Heusch M, Lemaire MM, et al. IL-22 is required for imiquimod-induced psoriasiform skin inflammation in mice. J Immunol. 2012;188(1):462-469. 
37. Coban M, Tasli L, Turgut S, et al. Association of adipokines, insulin resistance, hypertension and dyslipidemia in patients with psoriasis vulgaris. Ann Dermatol. 2016;28(1):74-79.

38. Luo Y, Liu M. Adiponectin: a versatile player of innate immunity. J Mol Cell Biol. 2016;8(2):120-128.

39. Zhang LJ, Guerrero-Juarez CF, Hata T, et al. Dermal adipocytes protect against invasive Staphylococcus aureus skin infection. Science. 2015;347(6217):67-71.

40. McGonagle D, Benjamin M, Tan AL. The pathogenesis of psoriatic arthritis and associated nail disease: not autoimmune after all? Curr Opin Rheumatol. 2009;21(4):340-347.

41. Kruithof E, Baeten D, De Rycke L, et al. Synovial histopathology of psoriatic arthritis, both oligo-and polyarticular, resembles spondyloarthropathy more than it does rheumatoid arthritis. Arthritis Res Ther. 2005;7(3):R569-R580.

42. Macchi V, Porzionato A, Sarasin G, et al. The infrapatellar adipose body: a histotopographic study. Cell Tiss Organ. 2016;201(3):220-231.

43. Sbarbati A, Accorsi D, Benati D, et al. Subcutaneous adipose tissue classification. Eur J Histochem. 2010;54(4):e48.

44. Bertossi D, Conti G, Bernardi P, et al. Classification of fat pad of the third medium of the face. Aesth Med. 2015;1(3):103-109.

45. Steco C. Functional Atlas of the Human Fascial System. Philadelphia, PA: Elsevier Health Sciences; 2014.

46. Kruglikov IL. General theory of body contouring: 2. Modulation of mechanical properties of subcutaneous fat tissue. J Cosm Dermatol Sci Appl. 2014;4(1):117-127.

47. Khan T, Muise ES, Iyengar $P$, et al. Metabolic dysregulation and adipose tissue fibrosis: role of collagen VI. Mol Cell Biol. 2009;29(6):1575-1591.

48. Clockaerts S, Bastiaansen-Jenniskens YM, Runhaar J, et al. The infrapatellar fat pad should be considered as an active osteoarthritic joint tissue: a narrative review. Osteoarthr Cartil. 2010;18(7):876-882.

49. Klein-Wieringa IR, Kloppenburg M, Bastiaansen-Jenniskens YM, et al. The infrapatellar fat pad of patients with osteoarthritis has an inflammatory phenotype. Ann Rheum Dis. 2011;70(5):851-857.

50. Sudoł-Szopińska I, Kontny E, Zaniewicz-Kaniewska K, ProhorecSobieszek M, Saied F, Maśliński W. Role of inflammatory factors and adipose tissue in pathogenesis of rheumatoid arthritis and osteoarthritis. Part I: rheumatoid adipose tissue. J Ultrason. 2013;13(53):192-201.

51. Sudo1-Szopińska I, Hrycaj P, Prohorec-Sobieszek M. Role of inflammatory factors and adipose tissue in pathogenesis of rheumatoid arthritis and osteoarthritis. Part II: inflammatory background of osteoarthritis. J Ultrason. 2013;13(54):319-328.

52. Eymard F, Chevalier X. Inflammation of the patellar fat pad. Joint Bone Spine. 2016;83(4):389-393.

53. Han W, Cai S, Liu Z, et al. Infrapatellar fat pad in the knee: is local fat good or bad for knee osteoarthritis? Arth Res Ther. 2014;16(4):R145.

54. Cai J, Xu J, Wang K, et al. Association between infrapatellar fat pad volume and knee structural changes in patients with knee osteoarthritis. J Rheumatol. 2015;42(10):1878-1884.

55. Wollina U, Heinig B, Langner D, Nowak A. Juxta-articular adiposis dolorosa (Dercum's disease type IV): report of four cases and treatment by dermolipectomy. Wien Med Wochenschr. 2015;165(17-18): 374-377.

56. Falsetti P, Frediani B, Acciai C, Baldi F, Filippou G, Marcolongo R. Heel fat pad involvement in rheumatoid arthritis and in spondyloarthropathies: an ultrasonographic study. Scand J Rheumatol. 2004; 33(5):327-331.

57. Shaw HM, Santer RM, Watson AH, Benjamin M. Adipose tissue at entheses: the innervation and cell composition of the retromalleolar fat pad associated with the rat Achilles tendon. J Anat. 2007;211(4): 436-443.

58. Pingel J, Petersen MCH, Fredberg U, et al. Inflammatory and metabolic alterations of Kager's fat pad in chronic Achilles tendinopathy. PLoS One. 2015;10(5):e0127811.

59. Sudoł-Szopińska I, Kwiatkowska B, Prochorec-Sobieszek M, Maśliński W. Enthesopathies and enthesitis. Part 1. Etiopathogenesis. J Ultrason. 2015;15(60):72-84.
60. Neumann E, Junker S, Schett G, et al. Adipokines in bone disease. Nat Rev Rheumatol. 2016;12(5):296-302.

61. Conde J, Scotece M, López V, et al. Differential expression of adipokines in infrapatellar fat pad (IPFP) and synovium of osteoarthritis patients and healthy individuals. Ann Rheum Dis. 2014;73(3):631-633.

62. Kontny E, Plebanczyk M, Lisowska B, Olszewska M, Maldyk P, Maslinski W. Comparison of rheumatoid articular adipose and synovial tissue reactivity to proinflammatory stimuli: contribution to adipocytokine network. Ann Rheum Dis. 2012;71(2):262-267.

63. Gandhi R, Takahashi M, Virtanen C, Syed K, Davey JR, Mahomed NN. Microarray analysis of the infrapatellar fat pad in knee osteoarthritis: relationship with joint inflammation. J Rheumatol. 2011;38(9): 1966-1972.

64. Conde J, Scotece M, Abella V, et al. Identification of novel adipokines in the joint. Differential expression in healthy and osteoarthritis tissues. PLoS One. 2015;10(4):e0123601

65. Hammarstedt A, Hedjazifar S, Jenndahl L, et al. WISP2 regulates preadipocyte commitment and PPAR $\gamma$ activation by BMP4. Proc Nat Acad Sci US A. 2013;110(7):2563-2568.

66. Chen TH, Chen L, Hsieh MS, Chang CP, Chou DT, Tsai SH. Evidence for a protective role for adiponectin in osteoarthritis. Biochim Biophys Acta. 2006;1762(8):711-718.

67. Honsawek S, Chayanupatkul M. Correlation of plasma and synovial fluid adiponectin with knee osteoarthritis severity. Arch Med Res. 2010;41(8):593-598.

68. Conde J, Scotece M, López V, et al. Adipokines: novel players in rheumatic diseases. Discov Med. 2013;15(81):73-83.

69. Hoffmann MH, Bruns H, Bäckdahl L, et al. The cathelicidins LL-37 and rCRAMP are associated with pathogenic events of arthritis in humans and rats. Ann Rheum Dis. 2013;72(7):1239-1248.

70. Kahlenberg JM, Kaplan MJ. Little peptide, big effects: the role of LL-37 in inflammation and autoimmune disease. J Immunol. 2013; 191(10):4895-4901.

71. Castelino M, Eyre S, Upton M, Ho P, Barton A. The bacterial skin microbiome in psoriatic arthritis, an unexplored link in pathogenesis: challenges and opportunities offered by recent technological advances. Rheumatology (Oxford). 2013;53(5):777-784.

72. Bierkarre H, Harder J, Cuthbert R, et al. Differential expression of antimicrobial peptides in psoriasis and psoriatic arthritis as a novel contributory mechanism for skin and joint disease heterogeneity. Scand J Rheumatol. 2016;45(3):188-196.

73. Peric M, Koglin S, Dombrowski Y, et al. Vitamin D analogs differentially control antimicrobial peptide/“alarmin” expression in psoriasis. PLoS One. 2009;4(7):e6340.

74. Reinholz M, Ruzicka T, Schauber J. Cathelicidin LL-37: an antimicrobial peptide with a role in inflammatory skin disease. Ann Dermatol. 2012;24(2):126-135.

75. Clausen ML, Slotved HC, Krogfelt KA, Andersen PS, Agner T. In vivo expression of antimicrobial peptides in atopic dermatitis. Exp Dermatol. 2016;25(1):3-9.

76. Miller LS, Cho JS. Immunity against Staphylococcus aureus cutaneous infections. Nat Rev Immunol. 2011;11(8):505-518.

77. Lowry MB, Guo C, Borregaard N, GombartAF. Regulation of the human cathelicidin antimicrobial peptide gene by $1 \alpha, 25$-dihydroxyvitamin D 3 in primary immune cells. J Steroid Biochem Mol Biol. 2014;143: 183-191.

78. Hoang-Yen Tran D, Hoang-Ngoc Tran D, Mattai SA, et al. Cathelicidin suppresses lipid accumulation and hepatic steatosis by inhibition of the CD36 receptor. Int J Obes (Lond). 2016;40(9):1424-1434.

79. Silverstein RL, Febbraio M. CD36, a scavenger receptor involved in immunity, metabolism, angiogenesis, and behavior. Sci Signal. 2009;2(72):re3.

80. Cescon M, Gaffazzo F, Chen P, Bonaldo P. Collagen VI at a glance. J Cell Sci. 2015;128(19):3525-3531.

81. Gara SK, Grumati P, Squarzoni S, et al. Differential and restricted expression of novel collagen VI chains in mouse. Matrix Biol. 2011; 30(4):248-257. 
82. Bonod-Bidaud $\mathrm{C}$, Roulet $\mathrm{M}$, Hansen $\mathrm{U}$, et al. In vivo evidence for a bridging role of a collagen $\mathrm{V}$ subtype at the epidermis-dermis interface. J Invest Dermatol. 2012;132(7):1841-1849.

83. Swindell WR, Remmer HA, Sarkar MK, et al. Proteogenomic analysis of psoriasis reveals discordant and concordant changes in mRNA and protein abundance. Genome Med. 2015;7(1):86.

84. Divoux A, Tordjman J, Lacasa D, et al. Fibrosis in human adipose tissue: composition, distribution, and link with lipid metabolism and fat mass loss. Diabetes. 2010;59(11):2817-2825.

85. Park J, Scherer PE. Adipocyte-derived endotrophin promotes malignant tumor progression. J Clin Invest. 2012;122(11):4243-4256.

86. Schnoor M, Cullen P, Lorkowski J, et al. Production of type VI collagen by human macrophages: a new dimension in macrophage functional heterogeneity. J Immunol. 2008;180(8):5707-5719.

87. Ezure T, Amano S. Negative regulation of dermal fibroblasts by enlarged adipocytes through release of free fatty acids. J Invest Dermatol. 2011;131(10):2004-2009.

88. Bober M, Enochsson C, Collin M, Mörgelin M. Collagen VI is a subepithelial adhesive target for human respiratory tract pathogens. $J$ Innate Immun. 2010;2(2):160-166.

89. Maaß T, Bayley CP, Mörgelin M, et al. Heterogeneity of collagen VI microfibrils: structural analysis of non-collagenous regions. $J$ Biol Chem. 2016;291(10):5247-5258.

90. Abdillahi SM, Balvanović S, Baumgarten M, et al. Collagen VI encodes antimicrobial activity: novel innate host defense properties of the extracellular matrix. J Innate Immun. 2012;4(4): 371-376.

91. Gesta S, Guntur K, Majumdar ID, et al. Reduced expression of collagen VI alpha 3 (COL6A3) confers resistance to inflammation-induced MCP1 expression in adipocytes. Obesity (Silver Spring). 2016; 24(8): 1695-1703.
92. Lackey DE, Burk DH, Ali MR, et al. Contributions of adipose tissue architectural and tensile properties toward defining healthy and unhealthy obesity. Am J Physiol Endocrinol Metab. 2014;306(3):E233-E246.

93. Braster Q, Silvestre-Roig C, Hartwig H, et al. Cathelicidin regulates myeloid cell accumulation in adipose tissue and promotes insulin resistance during obesity. Thromb Haemost. 2016;115(6):1237-1239.

94. Mok CC, Ko GTC, Ho LY, Yu KL, Chan PT, To CH. Prevalence of atherosclerotic risk factors and the metabolic syndrome in patients with chronic inflammatory arthritis. Arthritis Care Res (Hoboken). 2011;63(2): 195-202.

95. Ezure T, Amano S. Adiponectin and leptin up-regulate extracellular matrix production by dermal fibroblasts. Biofactors. 2007;31(3-4): 229-236.

96. Alexopoulos LG, Youn I, Bonaldo P, Guilak F. Developmental and osteoarthritic changes in Col6a1-knockout mice: biomechanics of type VI collagen in the cartilage pericellular matrix. Arthritis Rheum. 2009; 60(3):771-779.

97. Izu Y, Ansorge HL, Zhang G, et al. Dysfunctional tendon collagen fibrillogenesis in collagen VI null mice. Matrix Biol. 2011;30(1):53-61.

98. Kruglikov IL, Scherer PE. Dermal adipocytes and hair cycling: is spatial heterogeneity a characteristic feature of the dermal adipose tissue depot? Exp Dermatol. 2016;25(4):258-262.

99. Wilusz RE, Sanchez-Adams J, Guilak F. The structure and function of the pericellular matrix of articular cartilage. Matrix Biol. 2014;39:25-32.

100. Meyer LK, Ciaraldi TP, Henry RR, Wittgrove AC, Phillips SA. Adipose tissue depot and cell size dependency of adiponectin synthesis and secretion in human obesity. Adipocyte. 2013;2(4):217-226.

101. Hasegawa T, Sakamoto A, Wada A, Fukai T, Iida H, Ikeda S. Keratinocyte progenitor cells reside in human subcutaneous adipose tissue. PLoS One. 2015;10(2):e0118402.
Psoriasis: Targets and Therapy

\section{Publish your work in this journal}

Psoriasis: Targets and Therapy is international, peer-reviewed, open access journal focusing on psoriasis, nail psoriasis, psoriatic arthritis and related conditions, identification of therapeutic targets and the optimal use of integrated treatment interventions to achieve improved outcomes

\section{Dovepress}

and quality of life. Visit http://www.dovepress.com/testimonials.php to read real quotes from published authors. 